\title{
LPI Optimization Framework for Target Tracking in Radar Network Architectures Using Information-Theoretic Criteria
}

\author{
Chenguang Shi, ${ }^{1}$ Fei Wang, ${ }^{1}$ Mathini Sellathurai, ${ }^{2}$ and Jianjiang Zhou ${ }^{1}$ \\ ${ }^{1}$ Key Laboratory of Radar Imaging and Microwave Photonics, Ministry of Education, \\ Nanjing University of Aeronautics and Astronautics, No. 29, Yudao Street, Qinhuai District, Nanjing, Jiangsu 210016, China \\ ${ }^{2}$ School of Engineering and Physical Sciences, Heriot-Watt University, Edinburgh, UK \\ Correspondence should be addressed to Jianjiang Zhou; zjjee@nuaa.edu.cn
}

Received 26 February 2014; Revised 29 May 2014; Accepted 17 June 2014; Published 6 July 2014

Academic Editor: Shengqi Zhu

Copyright (C) 2014 Chenguang Shi et al. This is an open access article distributed under the Creative Commons Attribution License, which permits unrestricted use, distribution, and reproduction in any medium, provided the original work is properly cited.

\begin{abstract}
Widely distributed radar network architectures can provide significant performance improvement for target detection and localization. For a fixed radar network, the achievable target detection performance may go beyond a predetermined threshold with full transmitted power allocation, which is extremely vulnerable in modern electronic warfare. In this paper, we study the problem of low probability of intercept (LPI) design for radar network and propose two novel LPI optimization schemes based on information-theoretic criteria. For a predefined threshold of target detection, Schleher intercept factor is minimized by optimizing transmission power allocation among netted radars in the network. Due to the lack of analytical closed-form expression for receiver operation characteristics (ROC), we employ two information-theoretic criteria, namely, Bhattacharyya distance and J-divergence as the metrics for target detection performance. The resulting nonconvex and nonlinear LPI optimization problems associated with different information-theoretic criteria are cast under a unified framework, and the nonlinear programming based genetic algorithm (NPGA) is used to tackle the optimization problems in the framework. Numerical simulations demonstrate that our proposed LPI strategies are effective in enhancing the LPI performance for radar network.
\end{abstract}

\section{Introduction}

Radar network architecture, which is often called as distributed multiple-input multiple-output (MIMO) radar, has been recently put forward and is becoming an inevitable trend for future radar system design [1-3]. The performance of radar network heavily depends on optimal power allocation and transmission waveform design, so enhanced improvements on target detection and information extraction would be realized by spatial and signal diversities.

Currently, system design for target detection and information extraction performance improvement has been a long-term research topic in the distributed radar network literature. In [4], Fishler et al. propose the distributed MIMO radar concept and analyze the target detection performance for distributed MIMO radar. Yang and Blum in [5] study the target identification and classification for MIMO radar employing mutual information (MI) and the minimum mean-square error (MMSE) criteria. The authors in [6] investigate the problem of code design to improve the detection performance of multistatic radar in the presence of clutter. Niu et al. propose localization and tracking approaches for noncoherent MIMO radar, which provides significant performance enhancement over traditional phased array radar [7].

Power allocation problem in radar network architecture has been attracting contentiously growing attention, and some of the noteworthy publications include [8-14]. The work of [8] investigates the scheduling and power allocation problem in cognitive radar network for multiple-target tracking, in which an optimization criterion is proposed to find a suitable subset of antennas and optimal transmitted power allocation. Godrich et al. in [9-11] address the power allocation strategies for target localization in distributed multipleradar configurations and propose some performance driven resource allocation schemes. In [12], the authors investigate target threatening level based optimal power allocation for LPI radar network, where two effective algorithms are 
proposed to enhance the LPI performance for radar network. Furthermore, in $[13,14]$, several optimal power allocation algorithms for distributed MIMO radars with heterogeneous propagation losses are presented to enhance target detection and information extraction performance. However, up to now, the low probability of intercept (LPI) optimization for radar network architecture is still an open problem, which is playing an increasingly important role in modern electronic warfare $[1,15-18]$. Therefore, it is an urgent task to investigate the LPI optimization problem in radar network.

This paper will extend the results in [6] and propose two novel LPI optimization algorithms based on informationtheoretic criteria for radar network architecture. Our purpose is to minimize Schleher intercept factor by optimizing transmission power allocation among netted radars for a predefined threshold of target detection. Due to the lack of analytical closed-form expression for receiver operation characteristics (ROC), we employ two information-theoretic criteria including Bhattacharyya distance and J-divergence as the metrics for target detection performance. As demonstrated later, the proposed algorithms can provide significant LPI performance improvement for radar network. To the best of the authors' knowledge, no literature discussing the information-theoretic criteria based LPI optimization for radar network architecture was conducted prior to this work.

The remainder of this paper is organized as follows. Section 2 provides the radar network system model and binary hypothesis test. We first derive Schleher intercept factor for radar network in Section 3 and formulate the problems of information-theoretic criteria based LPI optimization, where the resulting nonconvex and nonlinear LPI optimization problems associated with different informationtheoretic criteria are cast under a unified framework and solved through the nonlinear programming based genetic algorithm (NPGA). Numerical examples are provided in Section 4. Finally, conclusion remarks are drawn in Section 5.

\section{System Model and the Optimal Detector}

2.1. Radar Network SNR Equation. We consider a radar network architecture with $N_{t}$ transmitters and $N_{r}$ receivers, which can be broken down into $N_{t} \times N_{r}$ transmitter-receiver pairs each with a bistatic component contributing to the entirety of the radar network signal-to-noise ratio (SNR) [1]. Depicted in Figure 1 is an example of $4 \times 4$ radar network. All the radars have acquired and are tracking the target with their directional antenna beams. The netted radars Radar1, Radar2, Radar3, and Radar4 transmit orthogonal waveforms (as solid lines) but receive and process all these echoes that are reflected from the target (as dotted lines) and send the estimates to one of the radars in the network for data fusion with data link.

For the radar network here, orthogonal polyphase codes are employed in the system, which have a large main lobeto-side lobe ratio. These codes have a more complicated signal structure making it more difficult to be intercepted and detected by a hostile intercept receiver.

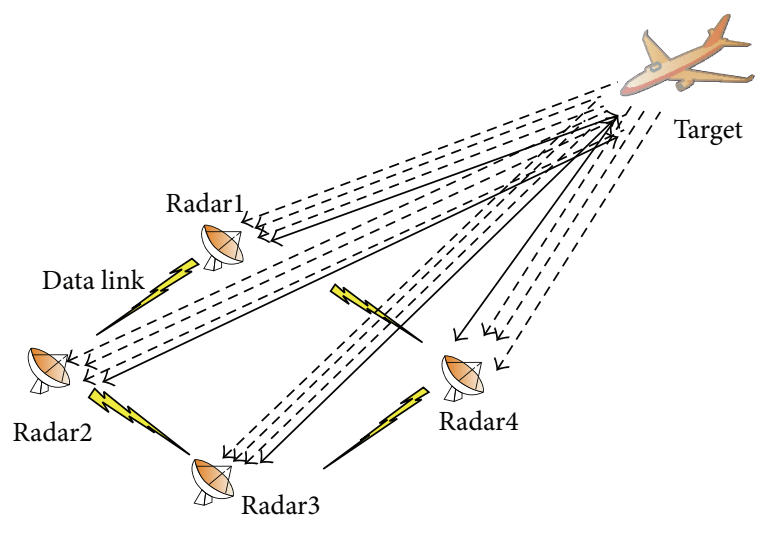

FIgURE 1: Example of an LPI radar network.

It is also supposed that the network system has a common precise knowledge of space and time. The radar network SNR can be calculated by summing up the SNR of each transmitreceive pair as [1] follows:

$$
\mathrm{SNR}_{\text {net }}=\sum_{i=1}^{N_{t}} \sum_{j=1}^{N_{r}} \frac{P_{t i} G_{t i} G_{r j} \sigma_{t i j} \lambda_{i}^{2}}{(4 \pi)^{3} k T_{o i j} B_{r i} F_{r j} R_{t i}^{2} R_{r j}^{2} L_{i j}}
$$

where the $P_{t i}$ is the $i$ th transmitter power, $G_{t i}$ is the $i$ th transmitting antenna gain, $G_{r j}$ is the $j$ th receiving antenna gain, $\sigma_{t i j}$ is the radar cross-section (RCS) of the target for the $i$ th transmitter and $j$ th receiver, $\lambda_{i}$ is the $i$ th transmitted wavelength, $k$ is Boltzmann's constant, $T_{o i j}$ is the receiving system noise temperature at the $j$ th receiver, $B_{r i}$ is the bandwidth of the matched filter for the $i$ th transmitted waveform, $F_{r j}$ is the noise factor for the $j$ th receiver, $L_{i j}$ is the system loss between the $i$ th transmitter and $j$ th receiver, $R_{t i}$ is the distance from the $i$ th transmitter to the target, and $R_{r j}$ is the distance from the target to the $j$ th receiver.

2.2. Radar Network Signal Model. According to the discussions in [14], the path gain contains the target reflection coefficient $g_{i j}$ and the propagation loss factor $p_{i j}$. Based on the central limit theorem, $g_{i j} \sim \mathrm{CN}\left(0, R_{g}\right)$, where $g_{i j}$ denotes the target reflection gain between radar $i$ and radar $j$. The propagation loss factor $p_{i j}$ is a function of radar antenna gain and waveform propagation distance, which is expressed as follows:

$$
p_{i j}=\frac{\sqrt{G_{t i} G_{r j}}}{R_{t i} R_{r j}} \text {. }
$$

It is supposed that the transmitted waveform of the $i$ th netted radar is $\sqrt{P_{t i}} x_{i}(t)$, and then the collected signals at the $j$ th receiver from a single point target can be written as follows:

$$
y_{j}(t)=\sum_{i=1}^{N_{t}} p_{i j} g_{i j} \sqrt{P_{t i}} x_{i}\left(t-\tau_{i j}\right)+n_{j}(t),
$$


where $\int\left|x_{j}(t)\right|^{2} d t=1, \tau_{i j}$ represents the time delay, $n_{j}(t)$ denotes the noise at receiver $j$, and the Doppler effect is negligible. At the $j$ th receiver, the received signal is matched filtered by time response $x_{k}^{*}(-t)$, and the output signal can be expressed as follows:

$$
\begin{aligned}
\tilde{y}_{j k}(t) & =\int y_{j}(t) \cdot x_{k}^{*}(\tau-t) d \tau \\
& =p_{j k} g_{j k} \sqrt{P_{t k}} \int x_{k}\left(\tau-\tau_{k j}\right) \cdot x_{k}^{*}(\tau-t) d \tau+\widetilde{n}_{j k}(t),
\end{aligned}
$$

where $\tilde{n}_{j k}(t)=\int n_{j}(\tau) \cdot x_{k}^{*}(\tau-t) d \tau$ and $\int x_{j}(\tau) \cdot x_{k}^{*}(\tau+t) d \tau=0$ for $k \neq j$.

The discrete time signal for the $j$ th receiver can be rewritten as follows:

$$
r_{j k} \triangleq \tilde{y}_{j k}\left(\tau_{j k}\right)=p_{j k} g_{j k} \sqrt{P_{t k}}+\theta_{j k}
$$

where $r_{k j}$ is the output of the matched filter at the receiver $j$ sampled at $\tau_{j k}, \theta_{j k}=\tilde{n}_{j k}\left(\tau_{j k}\right)$, and $\theta_{j k} \sim \mathrm{CN}\left(0, R_{\theta}\right)$. As mentioned before, we assume that all the netted radars have acquired and are tracking the target with their directional radar beams, and they transmit orthogonal waveforms while receiving and processing all these echoes that are reflected from the target. In this way, we can obtain $\tau_{j k}$.

2.3. Binary Hypothesis Test. With all the received signals, the target detection for radar network system leads to a binary hypothesis testing problem:

$$
\begin{aligned}
& H_{0}: r_{i j}=\theta_{i j} \\
& H_{1}: r_{i j}=p_{i j} g_{i j} \sqrt{P_{t i}}+\theta_{i j},
\end{aligned}
$$

where $1 \leq i \leq N_{t}, 1 \leq j \leq N_{r}$. The likelihood ratio test can be formulated as follows:

$$
\begin{aligned}
& H_{0}: T \triangleq \prod_{i=1}^{M} \prod_{j=1}^{N} \frac{f\left(r_{i j} \mid H_{1}\right)}{f\left(r_{i j} \mid H_{0}\right)}<\delta, \\
& H_{1}: T \triangleq \prod_{i=1}^{M} \prod_{j=1}^{N} \frac{f\left(r_{i j} \mid H_{1}\right)}{f\left(r_{i j} \mid H_{0}\right)}>\delta .
\end{aligned}
$$

As introduced in [14], the underlying detection problem can be equivalently rewritten as follows:

$$
\begin{aligned}
& H_{0}: r_{i j} \sim \mathrm{CN}\left(0, R_{\theta}\right), \\
& H_{1}: r_{i j} \sim \mathrm{CN}\left(0, R_{\theta}+P_{t i} R_{g} p_{i j}^{2}\right) .
\end{aligned}
$$

Then, we have the optimal detector as follows:

$$
\begin{aligned}
& H_{0}: T \triangleq \sum_{i=1}^{N_{t}} \sum_{j=1}^{N_{r}}\left|r_{i j}\right|^{2} \frac{2 P_{t i} p_{i j}^{2}}{R_{\theta}+P_{t i} R_{g} p_{i j}^{2}}<\delta, \\
& H_{1}: T \triangleq \sum_{i=1}^{N_{t}} \sum_{j=1}^{N_{r}}\left|r_{i j}\right|^{2} \frac{2 P_{t i} p_{i j}^{2}}{R_{\theta}+P_{t i} R_{g} p_{i j}^{2}}>\delta,
\end{aligned}
$$

where $\delta$ denotes the detection threshold.

\section{Problem Formulation}

In this section, we aim to obtain the optimal LPI performance for radar network architecture by judiciously designing the transmission power allocation among netted radars in the network. We first derive Schleher intercept factor for radar network system and then formulate the LPI optimization problems based on information-theoretic criteria. For a predefined threshold of target detection, Schleher intercept factor is minimized by optimizing transmission power allocation among netted radars. It is indicated in [6] that the analytical closed-form expression for ROC does not exist. As such, we resort to information-theoretic criteria, namely, Bhattacharyya distance and J-divergence. In what follows, the corresponding LPI optimization problems associated with different information-theoretic criteria are cast under a unified framework and can be solved conveniently through NPGA.

3.1. Schleher Intercept Factor for Radar Network. For radar network, it is supposed that all signals can be separately distinguished at every netted radar node. Assuming that every transmitter-receiver combination in the network can be the same and $R_{\text {net }}^{2} \triangleq R_{t i} \cdot R_{r j}$, in which case the radar network SNR equation (1) can be rewritten as follows (see Appendix A):

$$
\mathrm{SNR}_{\text {net }}=K_{\text {rad }} N_{r} \frac{P_{t}}{R_{\text {net }}^{4}},
$$

where

$$
K_{\mathrm{rad}}=\frac{G_{t} G_{r} \sigma_{t} \lambda^{2}}{(4 \pi)^{3} k T_{o} B_{r} F_{r} L},
$$

$P_{t}$ is the total transmitting power of radar network system.

Note that, when $N_{t}=N_{r}=1$, we can obtain the monostatic case

$$
\mathrm{SNR}_{\text {mon }}=K_{\text {rad }} \frac{P_{t}}{R_{\text {mon }}^{4}},
$$

where $R_{\text {mon }}$ is the distance between the monostatic radar and the target, while, for intercept receiver, the SNR equation is

$$
\mathrm{SNR}_{\mathrm{int}}=K_{\mathrm{int}} \frac{P_{t}}{R_{\mathrm{int}}^{2}}
$$




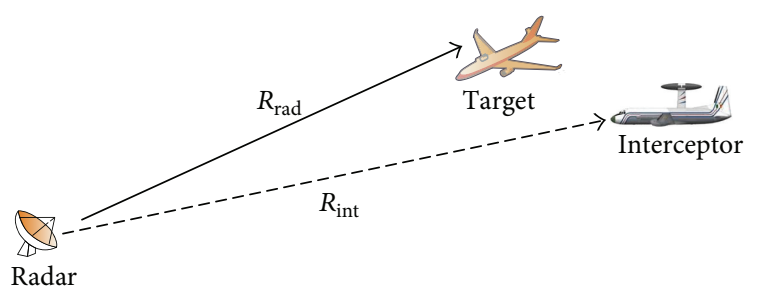

FIgURE 2: The geometry of radar, target, and interceptor.

where

$$
K_{\text {int }}=\frac{G_{t} G_{i} \lambda^{2}}{(4 \pi)^{2} k T_{o} B_{i} F_{i} L_{i}},
$$

$\mathrm{SNR}_{\text {int }}$ is the SNR at the interceptor signal processor input, $G_{t}^{\prime}$ is the gain of the radar's transmitting antenna in the direction of the intercept receiver, $G_{i}$ is the gain of the intercept receiver's antenna, $F_{i}$ is the interceptor noise factor, $B_{i}$ is the bandwidth of the interceptor, $R_{\text {int }}$ is the range from radar network to the intercept receiver, and $L_{i}$ refers to the losses from the radar antenna to the receiver. For simplicity, we assume that the intercept receiver is carried by the target. As such, the interceptor detects the radar emission from the main lobe; that is, $G_{t}^{\prime}=G_{t}$.

Herein, Schleher intercept factor is employed to evaluate LPI performance for radar network. The definition of Schleher intercept factor can be calculated as follows:

$$
\alpha=\frac{R_{\text {int }}}{R_{\text {rad }}},
$$

where $R_{\text {rad }}$ is the detection range of radar and $R_{\text {int }}$ is the intercept range of intercept receiver, as illustrated in Figure 2.

Based on the definition of Schleher intercept factor, if $\alpha>1$, radar can be detected by the interceptor, while if $\alpha \leq 1$, radar can detect the target and the interceptor cannot detect the radar. Therefore, radar can meet LPI performance when $\alpha \leq 1$. Moreover, minimization of Schleher intercept factor leads to better LPI performance for radar network architecture.

With the derivation of Schleher intercept factor in Appendix B, it can be observed that, for a predefined target detection performance, the closer the distance between radar system and target is, the less power the radar system needs to transmit on guarantee of target detection performance. For simplicity, the maximum intercept factor $\alpha_{\operatorname{mon}}^{\max }$ is normalized to be 1 when the monostatic radar transmits the maximal power $P_{\text {tot }}^{\max }$, and $\mathrm{SNR}_{\text {net }}=\mathrm{SNR}_{\text {mon }}$. Therefore, when the transmission power is $P_{t}$, the intercept factor for radar network system can be simplified as follows:

$$
\alpha_{\text {net }}=\frac{\alpha_{\text {mon }}}{N_{r}^{1 / 4}}=\left(\frac{P_{t}}{P_{\text {tot }}^{\max } \cdot N_{r}}\right)^{1 / 4},
$$

where $\alpha_{\text {mon }}$ is the Schleher intercept factor for monostatic radar. From (16), one can see that Schleher intercept factor $\alpha_{\text {net }}$ is reduced with the increase of the number of radar receivers $N_{r}$ and the decrease of the total transmission power $P_{t}$ in the network system.

\subsection{Information-Theoretic Criteria Based LPI Optimization}

3.2.1. Bhattacharyya Distance Based LPI Optimization Scheme. It is introduced in [6] that Bhattacharyya distance $B\left(p_{0}, p_{1}\right)$ measures the distance between two probability density functions (pdf) $p_{0}$ and $p_{1}$. The Bhattacharyya distance provides an upper bound on the probability of false alarm $P_{\mathrm{fa}}$ and at the same time yields a lower bound on the probability of detection $P_{d}$.

Consider two multivariate Gaussian distributions $P_{0}$ and $P_{1}, P_{0} \sim \mathrm{CN}\left(0, \sigma_{0}\right)$, and $P_{1} \sim \mathrm{CN}\left(0, \sigma_{1}\right)$; the Bhattacharyya distance $B\left(P_{0}, P_{1}\right)$ can be obtained as [6]

$$
B\left(P_{0}, P_{1}\right)=\log \left\{\frac{\operatorname{det}\left[0.5\left(\sigma_{0}+\sigma_{1}\right)\right]}{\sqrt{\operatorname{det}\left(\sigma_{0}\right) \operatorname{det}\left(\sigma_{1}\right)}}\right\} \text {. }
$$

Let $B\left[f\left(r \mid H_{0}\right), f\left(r \mid H_{1}\right)\right]$ represent Bhattacharyya distance between $H_{0}$ and $H_{1}$, where $f\left(r \mid H_{0}\right)$ and $f\left(r \mid H_{1}\right)$ are the pdfs of $\mathbf{r}$ under hypotheses $H_{0}$ and $H_{1}$. For the binary hypothesis testing problem, we have that

$$
\begin{aligned}
B_{\text {net }} & \triangleq B\left[f\left(r \mid H_{0}\right), f\left(r \mid H_{1}\right)\right] \\
& =\sum_{i=1}^{N_{t}} \sum_{j=1}^{N_{r}} \log \left(\frac{1+0.5 \zeta_{i j}}{\sqrt{1+\zeta_{i j}}}\right) \\
& =\sum_{i=1}^{N_{t}} \sum_{j=1}^{N_{r}} \log \left[\frac{1+P_{t i} R_{g} p_{i j}^{2}\left(2 R_{\theta}\right)^{-1}}{\sqrt{1+P_{t i} R_{g} p_{i j}^{2}\left(R_{\theta}\right)^{-1}}}\right] \\
& =\sum_{i=1}^{N_{t}} \sum_{j=1}^{N_{r}} \log \left[\frac{1+P_{t i} R_{g} G_{t i} G_{r j}\left(2 R_{\theta} R_{t i}^{2} R_{r j}^{2}\right)^{-1}}{\sqrt{1+P_{t i} R_{g} G_{t i} G_{r j}\left(R_{\theta} R_{t i}^{2} R_{r j}^{2}\right)^{-1}}}\right] .
\end{aligned}
$$

Based on the discussion in [6], maximization of the Bhattacharyya distance minimizes the upper bound on $P_{\mathrm{fa}}$ while it maximizes the lower bound on $P_{d}$. As expressed in (18), the Bhattacharyya distance derived here can be applied to evaluate the target detection performance of radar network as a function of different parameters, such as the transmitting power of each netted radar and the number of netted radars in the network. Intuitively, the greater the Bhattacharyya distance between the two distributions of the binary hypothesis testing problem, the better the capability of radar network system to detect the target, which would make the network system more vulnerable in modern electronic warfare. Therefore, the Bhattacharyya distance can provide guidance to the problem of LPI optimization for radar network architecture.

Here, we focus on the LPI optimization problem for radar network architecture, where Schleher intercept factor is minimized by optimizing transmission power allocation among netted radars in the network for a predetermined 
Bhattacharyya distance threshold, such that the LPI performance is met on the guarantee of target detection performance. Eventually, the underlying LPI optimization problem can be formulated as follows:

$$
\begin{array}{ll}
\underset{\overrightarrow{\mathbf{P}}_{\mathbf{t}}}{\min } & \alpha_{\text {net }}, \\
\text { s.t.: } & B_{\text {net }} \geq B^{\text {th }}, \\
& \sum_{i=1}^{N_{t}} P_{t i} \leq P_{\text {tot }}^{\max }, \\
& 0 \leq P_{t i} \leq P_{t i}^{\max } \quad(\forall i),
\end{array}
$$

where $\overrightarrow{\mathbf{P}}_{\mathbf{t}}=\left[P_{t 1}, P_{t 2}, \ldots, P_{t N_{t}}\right]^{T}$ is the transmitting power of radar network, $B^{\text {th }}$ is the Bhattacharyya distance threshold for target detection, $P_{\text {tot }}^{\max }$ is the maximum total transmission power of radar network, and $P_{t i}^{\max }$ (for all $i$ ) is the maximum transmission power of the corresponding netted radar node.

3.2.2. J-Divergence Based LPI Optimization Scheme. The Jdivergence $J\left(p_{0}, p_{1}\right)$ is another metric to measure the distance between two pdfs $p_{0}$ and $p_{1}$. It is defined as follows:

$$
J\left(p_{0}, p_{1}\right) \triangleq D\left(p_{0} \| p_{1}\right)+D\left(p_{1} \| p_{0}\right)
$$

where $D(\cdot)$ is the Kullback-Leibler divergence. It is shown in [19] that, for any fixed value of $P_{\mathrm{fa}}$,

$$
D\left[f\left(r \mid H_{0}\right) \| f\left(r \mid H_{1}\right)\right]=\lim _{N \rightarrow \infty}\left[-\frac{1}{N} \log \left(1-P_{d}\right)\right]
$$

and, for any fixed value of $P_{d}$, we can obtain

$$
D\left[f\left(r \mid H_{1}\right) \| f\left(r \mid H_{0}\right)\right]=\lim _{N \rightarrow \infty}\left[-\frac{1}{N} \log \left(P_{\mathrm{fa}}\right)\right] .
$$

From (21) and (22), we can observe that for any fixed $P_{\mathrm{fa}}$ the maximization of Kullback-Leibler divergence $D\left[f\left(r \mid H_{0}\right) \|\right.$ $\left.f\left(r \mid H_{1}\right)\right]$ results in an asymptotic maximization of $P_{d}$, while for any fixed $P_{d}$ the maximization of Kullback-Leibler divergence $D\left[f\left(r \mid H_{1}\right) \| f\left(r \mid H_{0}\right)\right]$ results in an asymptotic minimization of $P_{\mathrm{fa}}$.

With the derivation in [6], we have that

$$
\begin{aligned}
J_{\text {net }} & \triangleq J\left[f\left(r \mid H_{0}\right), f\left(r \mid H_{1}\right)\right] \\
& =\sum_{i=1}^{N_{t}} \sum_{j=1}^{N_{r}} \frac{\zeta_{i j}^{2}}{1+\zeta_{i j}}
\end{aligned}
$$

$$
\begin{aligned}
& =\sum_{i=1}^{N_{t}} \sum_{j=1}^{N_{r}} \frac{\left[P_{t i} R_{g} p_{i j}^{2}\left(R_{\theta}\right)^{-1}\right]^{2}}{1+P_{t i} R_{g} p_{i j}^{2}\left(R_{\theta}\right)^{-1}} \\
& =\sum_{i=1}^{N_{t}} \sum_{j=1}^{N_{r}} \frac{\left[P_{t i} R_{g} G_{t i} G_{r j}\left(R_{\theta} R_{t i}^{2} R_{r j}^{2}\right)^{-1}\right]^{2}}{1+P_{t i} R_{g} G_{t i} G_{r j}\left(R_{\theta} R_{t i}^{2} R_{r j}^{2}\right)^{-1}} .
\end{aligned}
$$

Consequently, the corresponding LPI optimization problem can be expressed as follows:

$$
\begin{array}{ll}
\underset{\vec{P}_{t}}{\min } & \alpha_{\text {net }}, \\
\text { s.t.: } & J_{\text {net }} \geq J^{\text {th }}, \\
& \sum_{i=1}^{N_{t}} P_{t i} \leq P_{\text {tot }}^{\max }, \\
& 0 \leq P_{t i} \leq P_{t i}^{\max } \quad(\forall i),
\end{array}
$$

where $J$ th is the J-divergence threshold for target detection.

3.3. The Unified Framework Based on NPGA. In this subsection, we cast the LPI optimization problems based on various information-theoretic criteria investigated earlier under a unified optimization framework. Furthermore, we formulate the following general form of the optimization problems in (19) and (24):

$$
\begin{array}{ll}
\min _{\vec{P}_{t}} & \alpha_{\text {net }}, \\
\text { s.t.: } & \gamma_{\text {net }} \geq \gamma^{\text {th }}, \\
& \sum_{i=1}^{N_{t}} P_{t i} \leq P_{\text {tot }}^{\max }, \\
& 0 \leq P_{t i} \leq P_{t i}^{\max } \quad(\forall i),
\end{array}
$$

where $\gamma_{\text {net }} \in\left\{B_{\text {net }}, J_{\text {net }}\right\}$ and $\gamma^{\text {th }}$ is the corresponding threshold for target detection.

In this paper, we utilize the nonlinear programming based genetic algorithm (NPGA) to seek the optimal solutions to the resulting nonconvex, nonlinear, and constrained problem (25). The NPGA has a good performance on the convergence speed, and it improves the searching performance of ordinary genetic algorithm.

The NPGA procedure is illustrated in Figure 3, where the population initialization module is utilized to initialize the population according to the resulting problem, while the calculating fitness value module is to calculate the fitness values of individuals in the population. Selection, crossover, and mutation are employed to seek the optimal solution, where $N$ is a constant. If the evolution is $N$ 's multiples, we can use NP approach to accelerate the convergence speed.

So far, we have completed the derivation of Schleher intercept factor for radar network architecture and 


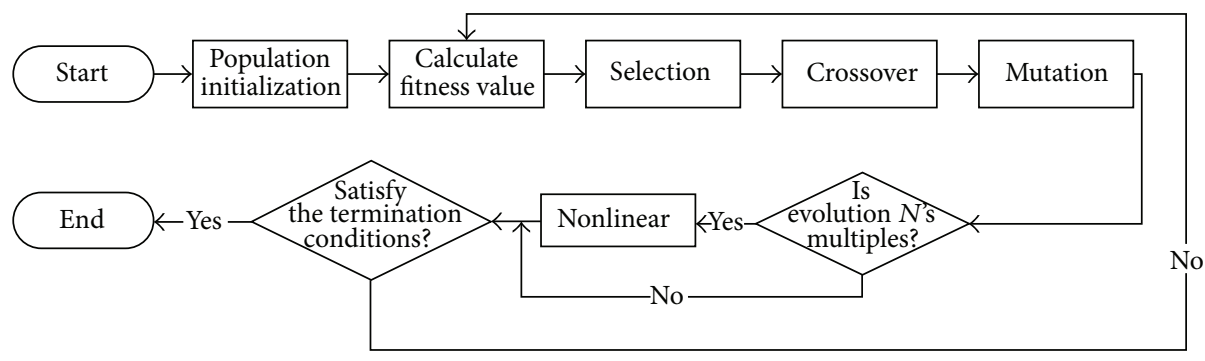

FIGURE 3: Flow diagram of NPGA procedure.

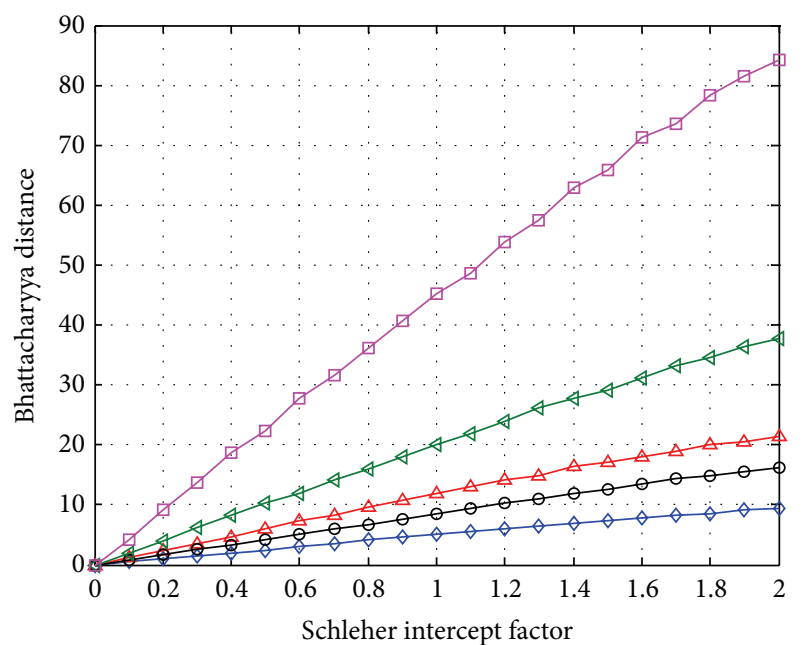

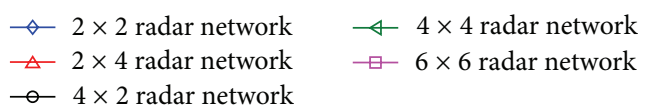

FIGURE 4: Bhattacharyya distance versus Schleher intercept factor for different radar network architectures.

the information-theoretic criteria based LPI optimization schemes. In what follows, some numerical simulations are provided to confirm the effectiveness of our presented LPI optimization algorithms for radar network architecture.

\section{Numerical Simulations}

In this section, we provide several numerical simulations to examine the performance of the proposed LPI optimization algorithms as (19) and (24). Throughout this section, we assume that $P_{\text {tot }}^{\max }=\sum_{i=1}^{N_{t}} P_{t i}=24 \mathrm{KW}, G_{t}=G_{r}=30 \mathrm{~dB}$, $R_{\theta}=10^{-10}$, and $R_{g}=1$. The SNR is set to be $13 \mathrm{~dB}$. The traditional monostatic radar can detect the target whose RCS is $0.05 \mathrm{~m}^{2}$ in the distance $R_{R \mathrm{MAX}}=106.1 \mathrm{~km}$ by transmitting the maximum power $P_{\text {tot }}^{\max }=24 \mathrm{KW}$, where the intercept factor is normalized to be 1 for simplicity.

4.1. LPI Performance Analysis. Figures 4 and 6 show the Bhattacharyya distance and logarithmic J-divergence versus Schleher intercept factor for different radar network architectures, respectively, which are conducted $10^{6}$ Monte

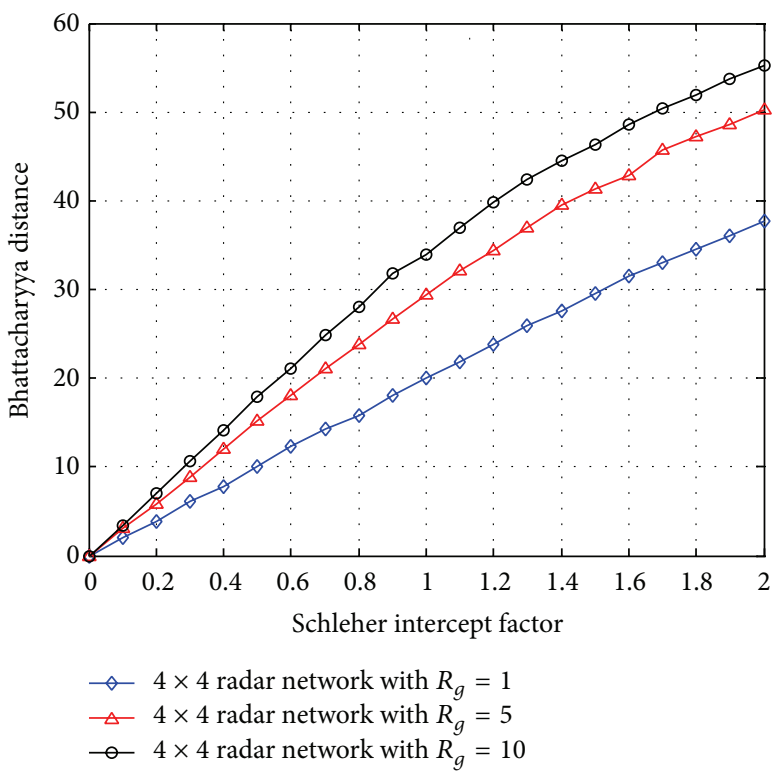

FIGURE 5: Bhattacharyya distance versus Schleher intercept factor for different target scattering intensity with $N_{t}=N_{r}=4$.

Carlo trials. We can observe in Figures 4 and 6 that as Schleher intercept factor increases from $\alpha_{\text {net }}=0$ to $\alpha_{\text {net }}=$ 2 the achievable Bhattacharyya distance and logarithmic Jdivergence are increased. This is due to the fact that as the intercept factor increases more transmission power would be allocated, which makes the achievable Bhattacharyya distance and logarithmic J-divergence increase correspondingly as theoretically proved in (18) and (23). Furthermore, it can be seen from Figures 4 and 6 that, with the same target detection threshold, Schleher intercept factor can be significantly reduced as the number of transmitters and receivers in the network system increases. Therefore, increasing the number of netted radars can effectively improve the LPI performance for radar network. This confirms the LPI benefits of the radar network architecture with more netted radars.

As shown in Figures 5 and 7, we illustrate the Bhattacharyya distance and logarithmic J-divergence versus Schleher intercept factor for different target scattering intensity with $N_{t}=N_{r}=4$, respectively. It is depicted that as the target scattering intensity increases from $R_{g}=1$ to $R_{g}=10$ the achievable Bhattacharyya distance and logarithmic J-divergence are significantly increased. This is 


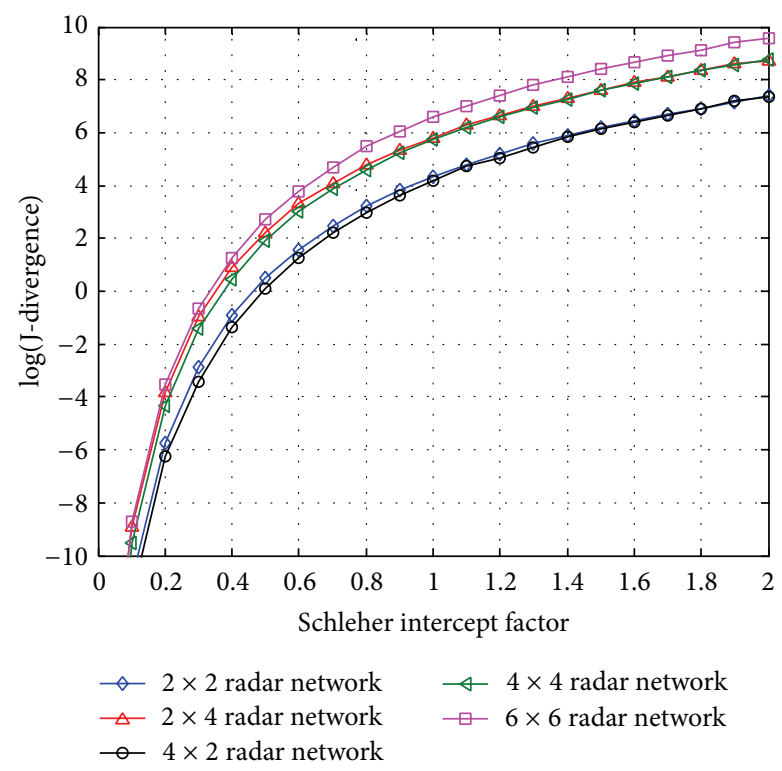

FIGURE 6: Logarithmic J-divergence versus Schleher intercept factor for different radar network architectures.

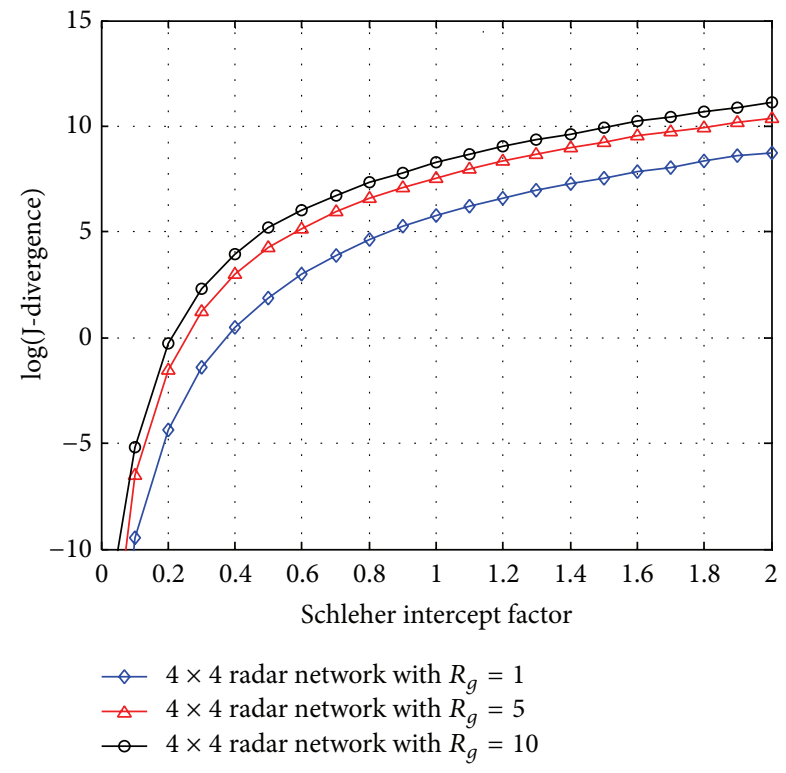

FIGURE 7: Logarithmic J-divergence versus Schleher intercept factor for different target scattering intensity with $N_{t}=N_{r}=4$.

because the radar network system can detect the target with large scattering intensity easily with high $P_{d}$ and low $P_{\text {fa }}$.

4.2. Target Tracking with LPI Optimization. In this subsection, we consider a $4 \times 4$ radar network system $\left(N_{t}=\right.$ $\left.N_{r}=4\right)$ in the simulation, and it is widely deployed in modern battlefield. The target detection threshold $\gamma^{\text {th }}$ can be calculated in the condition that the transmission power of each radar is $6 \mathrm{KW}$ in the distance $150 \mathrm{~km}$ between the radar network and the target, which is the minimum value of the basic performance requirement for target detection. As

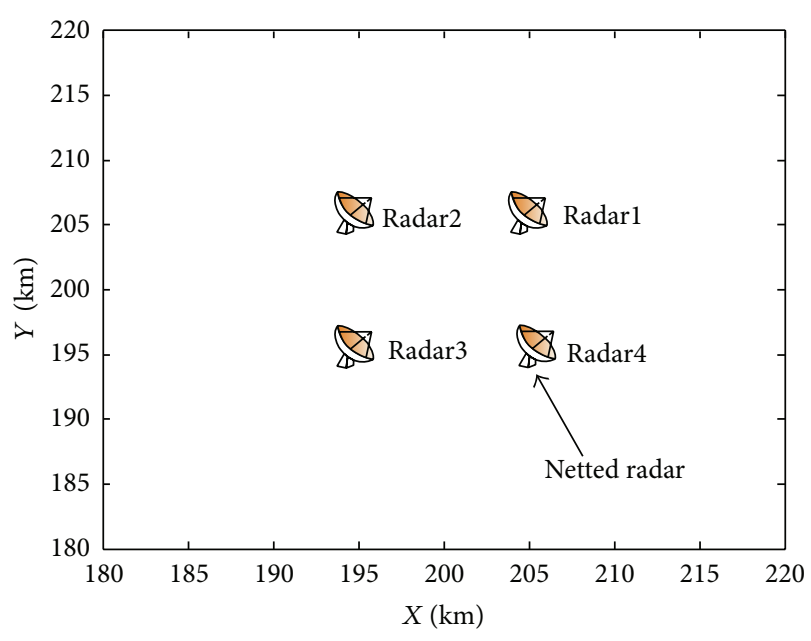

FIGURE 8: The radar network system configuration in two dimensions.

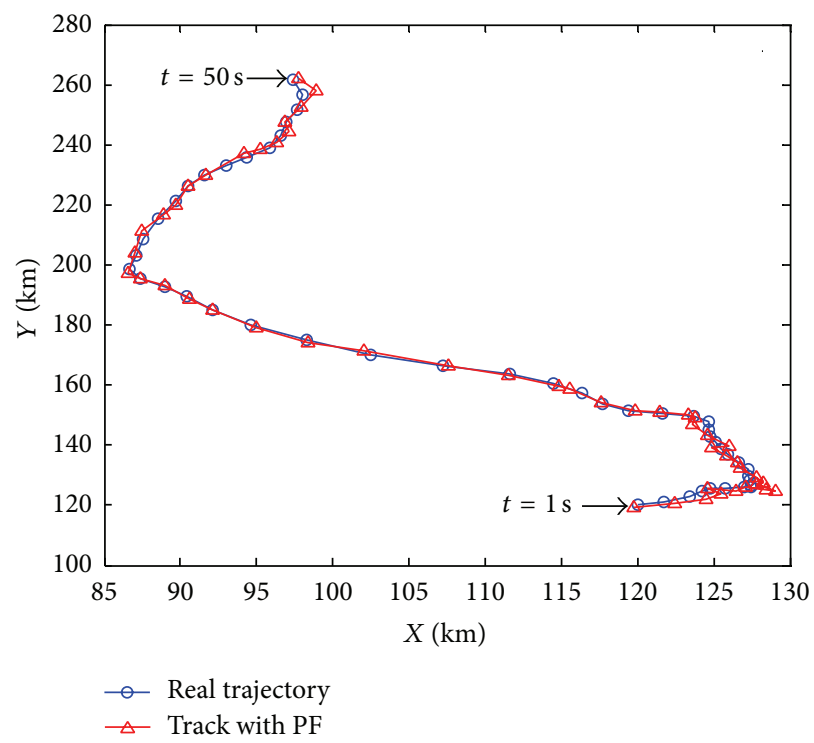

FIGURE 9: Target tracking scenario.

mentioned before, it is supposed that the intercept receiver is carried by the target. It is depicted in Figure 8 that the netted radars in the network are spatially distributed in the surveillance area at the initial time $t=0$.

We track a single target by utilizing particle filtering $(\mathrm{PF})$ method, where 5000 particles are used to estimate the target state. Figure 9 shows one realization of the target trajectory for $50 \mathrm{~s}$, and the tracking interval is chosen to be $1 \mathrm{~s}$. With the radar network configuration in Figure 8 and the target tracking scenario in Figure 9, we can obtain the distances changing curve between the netted radars and the target in the tracking process as depicted in Figure 10. Without loss of generality, we set Radar 1 as the distributed data fusion center and capitalize the weighted average approach to obtain the estimated target state. 


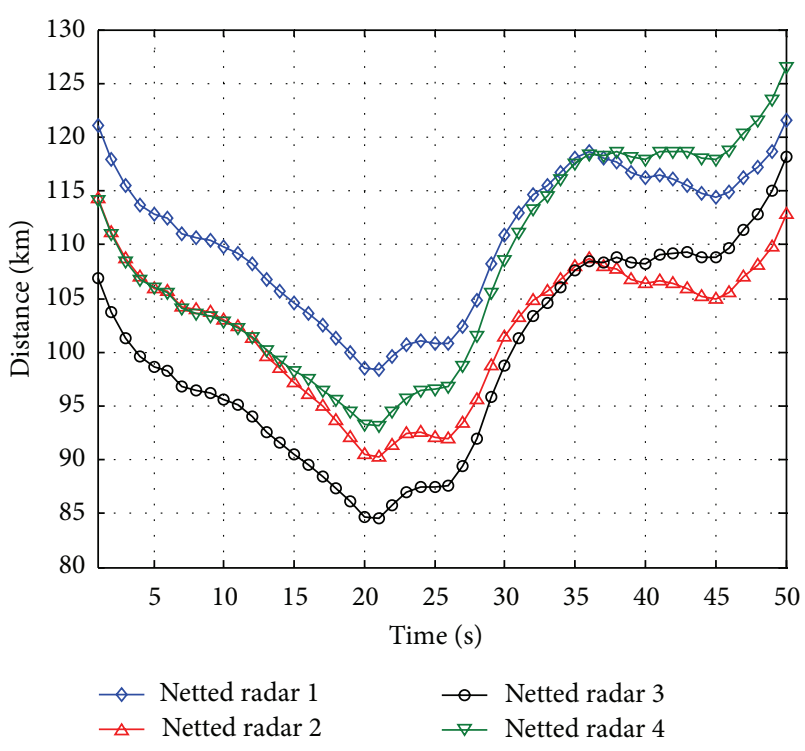

FIGURE 10: The distances between the netted radars and the target.

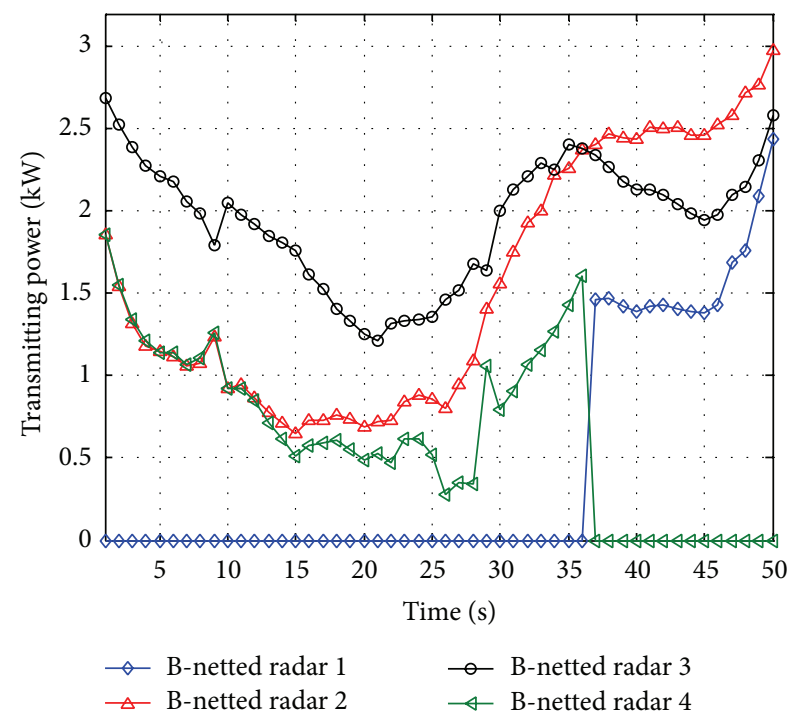

FIGURE 11: The transmitting power of netted radars utilizing Bhattacharyya distance based LPI optimization in the tracking process.

To obtain the optimal transmission power allocation of radar network, we utilize NPGA to solve (19) and (24). Let the population size be 100 , let the crossover probability be 0.6 , and let the mutation probability be 0.01 . The population evolves 10 generations. Figure 11 shows the transmitting power of netted radars utilizing Bhattacharyya distance based LPI optimization in the tracking process, while Figure 12 depicts the J-divergence based case. Before $t=36 \mathrm{~s}$, netted radars 2, 3, and 4 are selected to track the target, which are the ones closest to the target, while netted radar 1 is selected instead of radar 2 after $t=36 \mathrm{~s}$, which is because netted radars 1,2 , and 3 have the best channel conditions in the network. From Figures 11 and 12, we can see that the transmission power allocation is determined by the locations of single

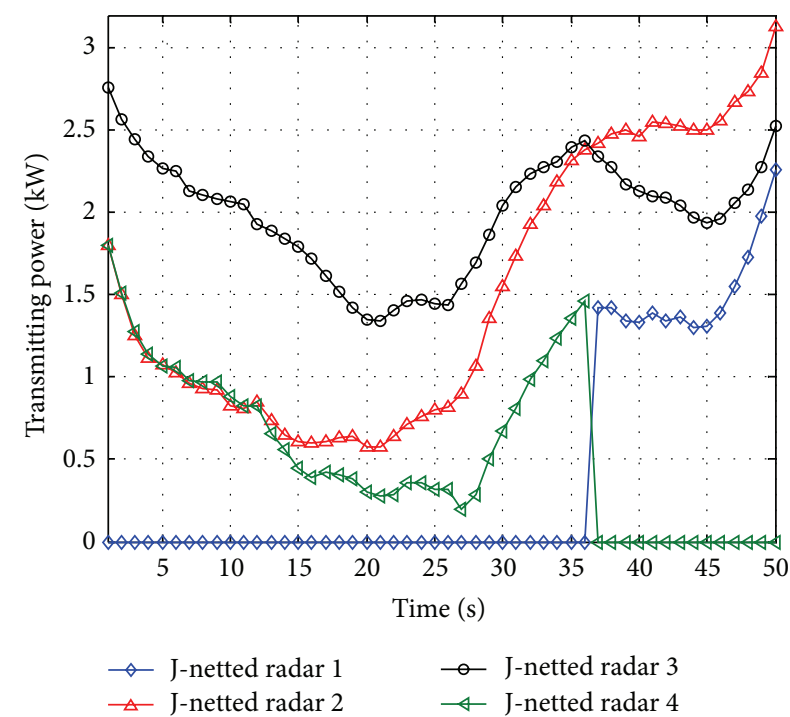

FIGURE 12: The transmitting power of netted radars utilizing Jdivergence based LPI optimization in the tracking process.

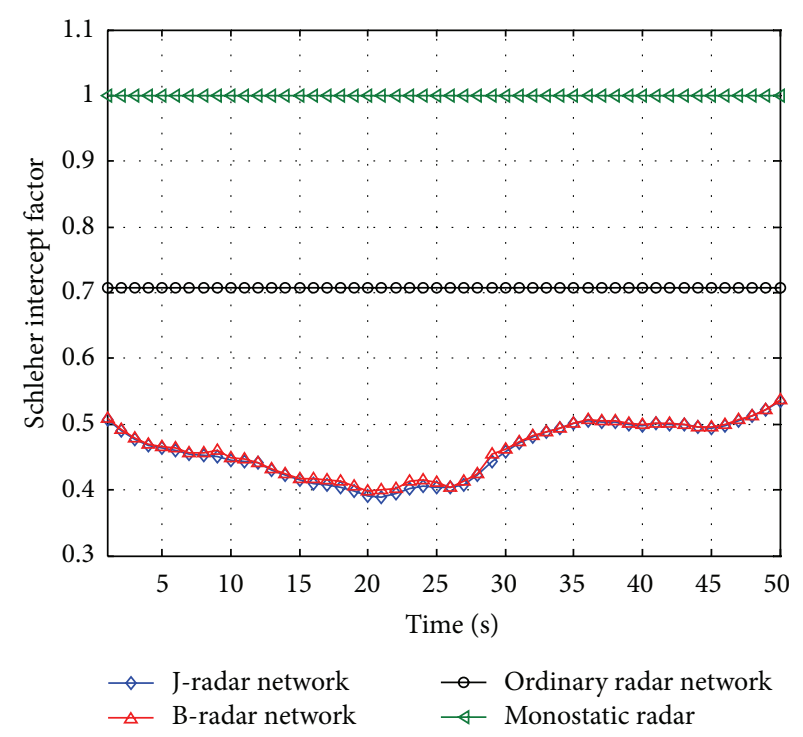

FIGURE 13: The normalized Schleher intercept factor comparison in the tracking process.

target relative to the netted radars and their propagation losses. To be specific, in the LPI optimization process, more transmitting power is allocated to the radar nodes that are located closer to the target; this is due to the fact that they suffer less propagation losses.

Figure 13 demonstrates the advantage of our proposed optimization problems based on information-theoretic criteria. The traditional monostatic radar transmits $24 \mathrm{KW}$ constantly, while the ordinary radar network has a constant sum of transmitted power $24 \mathrm{KW}$ and each radar node transmits uniform power. One can see that Schleher intercept factor for radar network employing the information-theoretic criteria based LPI optimization strategies is strictly smaller 
than that of traditional monostatic radar and ordinary radar network across the whole region, which further shows the LPI enhancement by exploiting our presented LPI optimization schemes in radar network to defend against passive intercept receiver. Moreover, it can be seen in Figure 13 that, in terms of the same system constraints and fundamental quantity, Bhattacharyya distance based LPI optimization is asymptotically equivalent to the J-divergence based case.

4.3. Discussion. According to Figures 4-13, we can deduce the following conclusions for radar network architecture.

(1) From Figures 4 to 7 , we can observe that as the predefined threshold of target detection increases more transmission power would be allocated for radar network to meet the detection performance, while the intercept factor is increased subsequently, which is vulnerable in electronic warfare. In other words, there exists a tradeoff between LPI and target detection performance in radar network system, and the LPI performance would be sacrificed with target detection consideration.

(2) In the numerical simulations, we observe that the proposed optimization schemes (19) and (24) can be employed to enhance the LPI performance for radar network. Based on the netted radars' spatial distribution with respect to the target, we can improve the LPI performance by optimizing transmission power allocation among netted radars. As indicated in Figures 11 and 12, netted radars with better channel conditions are favorable over others. In addition, it can be observed that exploiting our proposed algorithms can effectively improve the LPI performance of radar network to defend against intercept receiver, and Bhattacharyya distance based LPI optimization algorithm is asymptotically equivalent to the J-divergence based case under the same system constraints and fundamental quantity.

\section{Conclusions}

In this paper, we investigated the problem of LPI design in radar network architecture, where two LPI optimization schemes based on information-theoretic criteria have been proposed. The NPGA was employed to tackle the highly nonconvex and nonlinear optimization problems. Simulations have demonstrated that our proposed strategies are effective and valuable to improve the LPI performance for radar network, and it is indicated that these two optimization problems are asymptotically equivalent to each other under the same system constraints. Note that only single target was considered in this paper. Nevertheless, it is convenient to be extended to multiple targets scenario, and the conclusions obtained in this study suggest that similar LPI benefits would be obtained for the multiple targets case. Future work will look into the adaptive threshold design of target detection performance in radar network architectures.

\section{Appendices}

\section{A.}

Assume that every transmitter-receiver combination in the network can be the same and $R_{\text {net }}^{2} \triangleq R_{t i} \cdot R_{r j}$, where the radar network SNR (1) can be written as follows:

$$
\mathrm{SNR}_{\mathrm{net}}=K_{\mathrm{rad}} \sum_{i=1}^{N_{t}} \sum_{j=1}^{N_{r}} \frac{P_{t i}}{R_{\mathrm{net}}^{4}},
$$

where

$$
K_{\mathrm{rad}}=\frac{G_{t} G_{r} \sigma_{t} \lambda^{2}}{(4 \pi)^{3} k T_{o} B_{r} F_{r} L} .
$$

Assume that the sum of the effective radiated power (ERP) from all the radars in the network is equivalent to that of monostatic radar; that is,

$$
\mathrm{ERP}=\sum_{i=1}^{N_{t}} P_{t i} G_{t i}=P_{t} G_{t},
$$

where $P_{t}$ and $G_{t}$ are the transmitting power and transmitting antenna gain of the monostatic radar, respectively. For $G_{t i}=$ $G_{t}$ (for all $i$ ), we can rewrite (A.1) as follows:

$$
\mathrm{SNR}_{\text {net }}=K_{\text {rad }} N_{t} \frac{P_{t}}{R_{\text {net }}^{4}} .
$$

B.

According to (15), we can derive the intercept factor for radar network as

$$
\alpha_{\text {net }}=\frac{R_{\text {int }}}{R_{\text {net }}}=\left(\frac{P_{t} \cdot K_{\mathrm{int}}^{2} \cdot \mathrm{SNR}_{\mathrm{net}}}{K_{\mathrm{rad}} \cdot \mathrm{N}_{r} \cdot \mathrm{SNR}_{\mathrm{int}}^{2}}\right)^{1 / 4}
$$

and the intercept factor for conventional monostatic radar as

$$
\alpha_{\text {mon }}=\frac{R_{\text {int }}}{R_{\text {mon }}}=\left(\frac{P_{t} \cdot K_{\mathrm{int}}^{2} \cdot \mathrm{SNR}_{\mathrm{mon}}}{K_{\mathrm{rad}} \cdot \mathrm{SNR}_{\mathrm{int}}^{2}}\right)^{1 / 4} .
$$

When $\mathrm{SNR}_{\text {net }}=\mathrm{SNR}_{\text {mon }}$, we can readily obtain the relationship between the intercept factors for radar network $\alpha_{\text {net }}$ and for the monostatic case $\alpha_{\text {mon }}$ :

$$
\alpha_{\text {net }}=\frac{\alpha_{\text {mon }}}{N_{r}^{1 / 4}}
$$

Furthermore, for monostatic radar, we can assume that

$$
\mathrm{SNR}_{\text {mon }}=K_{\text {rad }} \frac{P_{t}}{R_{\text {mon }}^{4}}=K_{\text {rad }} \frac{P_{\text {tot }}^{\text {max }}}{R_{\text {RMAX }}^{4}},
$$

where $P_{\text {tot }}^{\max }$ is the maximal power of the monostatic rada$\mathrm{r}$ and $R_{R \mathrm{MAX}}$ is the corresponding maximal detection range. Then, we can obtain

$$
\frac{R_{\text {mon }}}{R_{R \text { MAX }}}=\left(\frac{P_{t}}{P_{\text {tot }}^{\max }}\right)^{1 / 4} .
$$


Similarly, for intercept receiver, we have the following:

$$
\begin{gathered}
\mathrm{SNR}_{\text {int }}=K_{\text {int }} \frac{P_{t}}{R_{\text {int }}^{2}}=K_{\text {int }} \frac{P_{\text {tot }}^{\max }}{R_{I M A X}^{2}}, \\
\frac{R_{\text {int }}}{R_{\text {IMAX }}}=\left(\frac{P_{t}}{P_{\text {tot }}^{\max }}\right)^{1 / 2},
\end{gathered}
$$

where $R_{\text {IMAX }}$ is the intercept range when the transmitting power of radar is $P_{\text {tot }}^{\max }$.

Using (15), (B.5), and (B.7), we can obtain the following expression:

$$
\frac{\alpha_{\mathrm{mon}}}{\alpha_{\mathrm{mon}}^{\max }}=\frac{R_{\mathrm{int}} / R_{\mathrm{mon}}}{R_{I \mathrm{MAX}} / R_{R \mathrm{MAX}}}=\left(\frac{P_{t}}{P_{\mathrm{tot}}^{\max }}\right)^{1 / 4},
$$

where $\alpha_{\operatorname{mon}}^{\max }$ is Schleher intercept factor corresponding to the maximal transmitting power $P_{\text {tot }}^{\max }$.

\section{Conflict of Interests}

The authors declare that there is no conflict of interests regarding the publication of this paper.

\section{Acknowledgments}

The authors would like to thank the anonymous reviewers for their comments that help to improve the quality of this paper. The support provided by the National Natural Science Foundation of China (Grant no. 61371170), Funding of Jiangsu Innovation Program for Graduate Education (CXLX13_154), the Fundamental Research Funds for the Central Universities, the Priority Academic Program Development of Jiangsu Higher Education Institutions (PADA), and Key Laboratory of Radar Imaging and Microwave Photonics, Ministry of Education, Nanjing University of Aeronautics and Astronautics, Nanjing, China, is gratefully acknowledged.

\section{References}

[1] E. P. Phillip, Detecting and Classifying Low Probability of Intercept Radar, Artech House, Boston, Mass, USA, 2009.

[2] A. M. Haimovich, R. S. Blum, and L. J. Cimini Jr., "MIMO radar with widely separated antennas," IEEE Signal Processing Magazine, vol. 25, no. 1, pp. 116-129, 2008.

[3] A. L. Hume and C. J. Baker, "Netted radar sensing," in Proceedings of the 2001 CIE International Conference on Radar, pp. 2326, 2001.

[4] E. Fishler, A. Haimovich, R. S. Blum, L. J. Cimini Jr., D. Chizhik, and R. A. Valenzuela, "Spatial diversity in radars-Models and detection performance," IEEE Transactions on Signal Processing, vol. 54, no. 3, pp. 823-838, 2006.

[5] Y. Yang and R. S. Blum, "MIMO radar waveform design based on mutual information and minimum mean-square error estimation," IEEE Transactions on Aerospace and Electronic Systems, vol. 43, no. 1, pp. 330-343, 2007.

[6] M. M. Naghsh, M. H. Mahmoud, S. P. Shahram, M. Soltanalian, and P. Stoica, "Unified optimization framework for multi-static radar code design using information-theoretic criteria," IEEE Transactions on Signal Processing, vol. 61, no. 21, pp. 5401-5416, 2013.
[7] R. Niu, R. S. Blum, P. K. Varshney, and A. L. Drozd, "Target localization and tracking in noncoherent multiple-input multiple-output radar systems," IEEE Transactions on Aerospace and Electronic Systems, vol. 48, no. 2, pp. 1466-1489, 2012.

[8] P. Chavali and A. Nehorai, "Scheduling and power allocation in a cognitive radar network for multiple-target tracking," IEEE Transactions on Signal Processing, vol. 60, no. 2, pp. 715-729, 2012.

[9] H. Godrich, A. Petropulu, and H. V. Poor, "Optimal power allocation in distributed multiple-radar configurations," in Proceeding of the IEEE International Conference on Acoustics, Speech, and Signal Processing (ICASSP '11), pp. 2492-2495, Prague, Czech Republic, May 2011.

[10] H. Godrich, A. P. Petropulu, and H. V. Poor, "Power allocation strategies for target localization in distributed multiple-radar architectures," IEEE Transactions on Signal Processing, vol. 59, no. 7, pp. 3226-3240, 2011.

[11] H. Godrich, A. Petropulu, and H. V. Poort, "Estimation performance and resource savings: tradeoffs in multiple radars systems," in Proceedings of the IEEE International Conference on Acoustics, Speech, and Signal Processing (ICASSP '12), pp. 52055208, Kyoto, Japan, March 2012.

[12] C. G. Shi, J. J. Zhou, F. Wang, and J. Chen, “Target threatening level based optimal power allocation for LPI radar network," in Proceedings of the 2nd International Symposium on Instrumentation and Measurement, Sensor Network and Automation (IMSNA '13), pp. 634-637, Toronto, Canada, December 2013.

[13] X. Song, P. Willett, and S. Zhou, "Optimal power allocation for MIMO radars with heterogeneous propagation losses," in Proceedings of the IEEE International Conference on Acoustics, Speech, and Signal Processing (ICASSP '12), pp. 2465-2468, Kyoto, Japan, March 2012.

[14] X. F. Song, P. Willett, S. Zhou, and J. Glaz, "MIMO radar detection with heterogeneous propagation losses," in Proceedings of the IEEE Statistical Signal Processing Workshop (SSP '12), pp. 776-779, August 2012.

[15] D. C. Schleher, "LPI radar: fact or fiction," IEEE Aerospace and Electronic Systems Magazine, vol. 21, no. 5, pp. 3-6, 2006.

[16] D. Lynch Jr., Introduction to RF Stealth, Sci Tech Publishing, 2004.

[17] A. G. Stove, A. L. Hume, and C. J. Baker, "Low probability of intercept radar strategies," IEE Proceedings Radar, Sonar and Navigation, vol. 151, no. 5, pp. 249-260, 2004.

[18] D. E. Lawrence, "Low probability of intercept antenna array beamforming," IEEE Transactions on Antennas and Propagation, vol. 58, no. 9, pp. 2858-2865, 2010.

[19] T. Kailath, "The divergence and Bhattacharyya distance measures in signal detection," IEEE Transaction on Communication Technology, vol. 15, no. 2, pp. 52-60, 1967. 

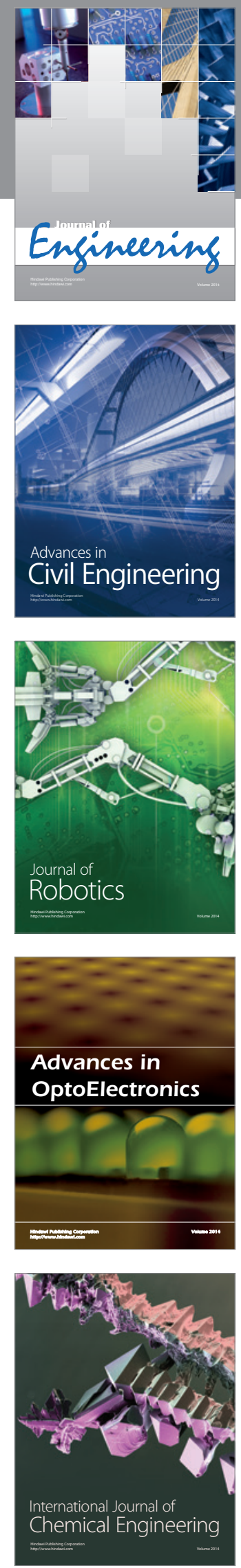

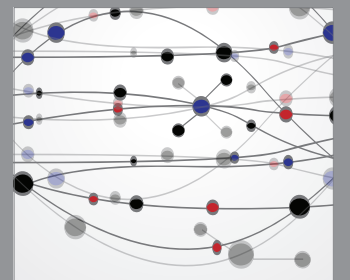

The Scientific World Journal
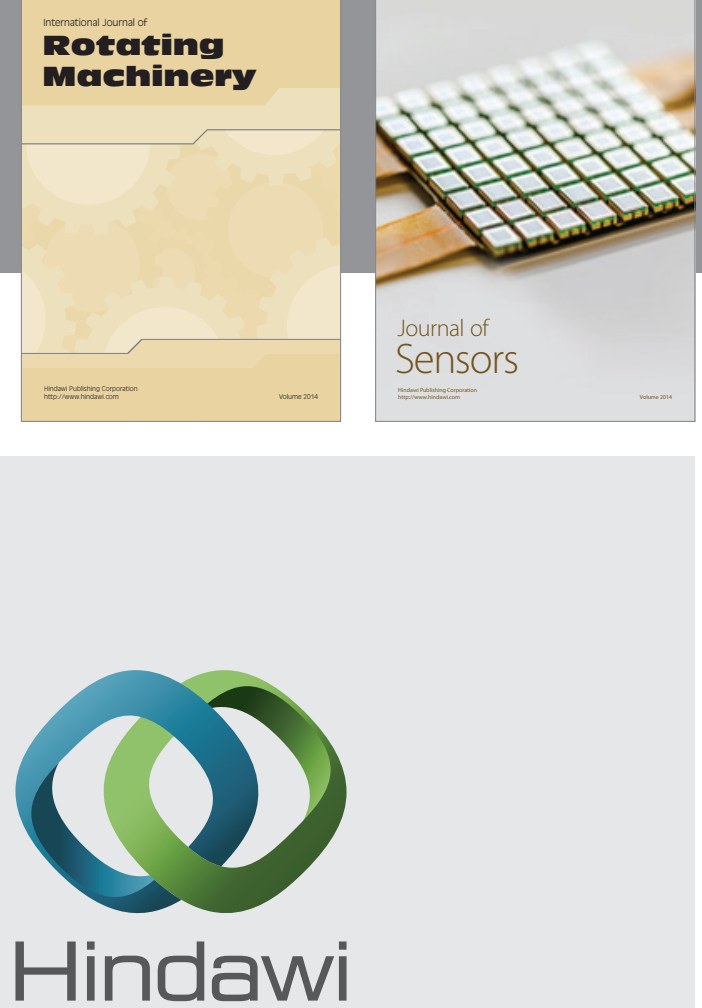

Submit your manuscripts at http://www.hindawi.com
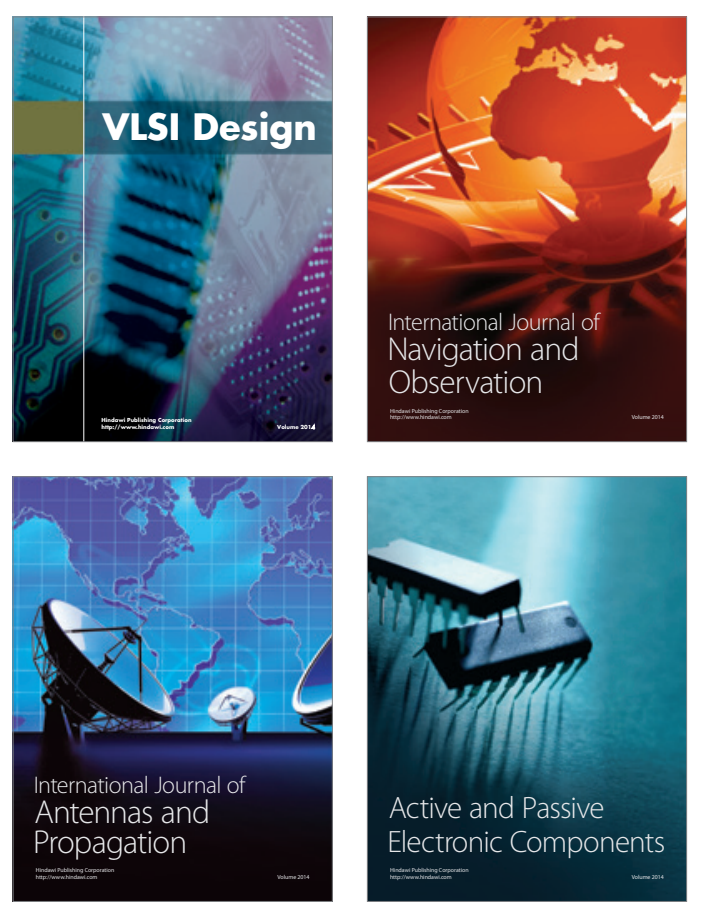
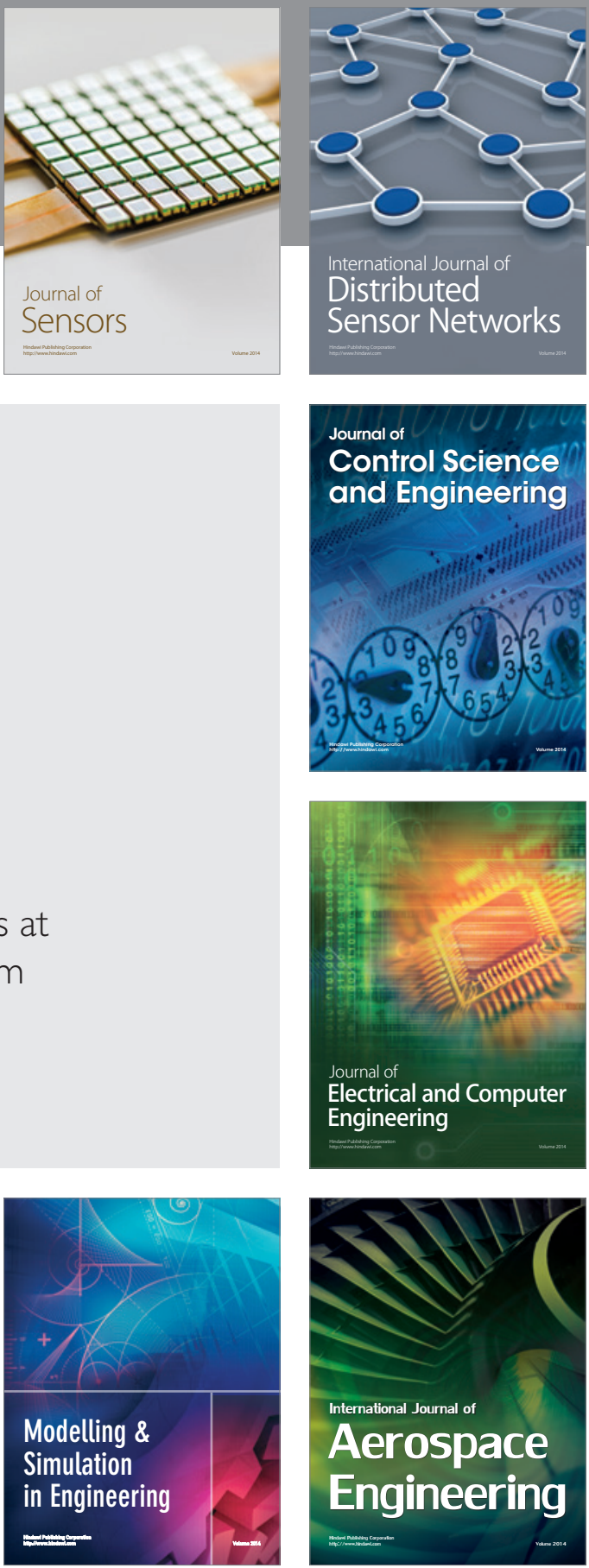

Journal of

Control Science

and Engineering
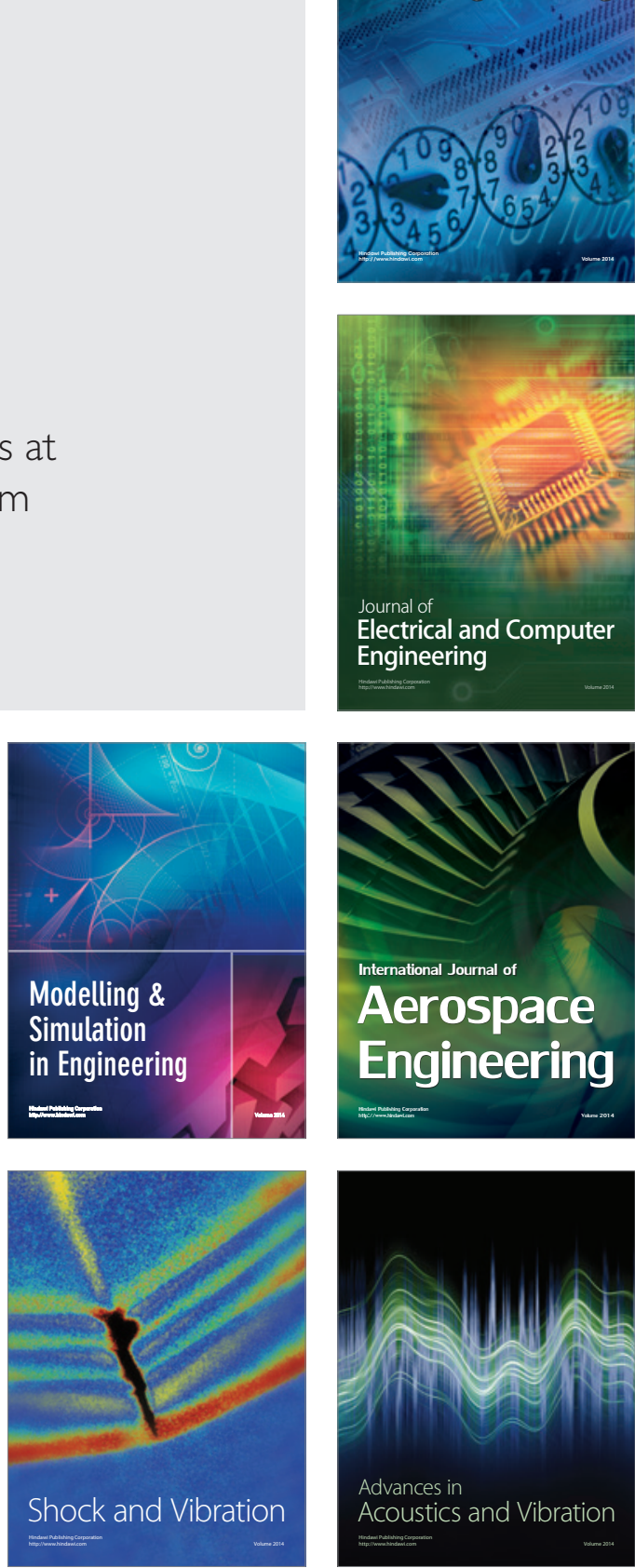